\title{
Difusión espacio-temporal de muertes por COVID-19 en Argentina
}

\author{
Carlos Marcelo Leveau ${ }^{1}$
}

Forma de citar

Leveau CM. Difusión espacio-temporal de muertes por COVID-19 en Argentina. Rev Panam Salud Publica. 2021;45:e3. https://doi.org/10.26633/RPSP.2021.3

Resumen

\begin{abstract}
Objetivos. Describir la difusión espacio-temporal de las muertes por COVID-19, y analizar sus desigualdades socio-espaciales en la Argentina.

Métodos. Se analizaron las muertes por COVID-19 ocurridas en Argentina al 17 de octubre de 2020 utilizando datos referidos al día, mes y año, y el lugar de residencia. Se utilizó la técnica de escaneo espacio-temporal por permutaciones para detectar la presencia de conglomerados espacio-temporales. Se compararon el nivel de pobreza, densidad poblacional y porcentaje de población adulta mayor entre las áreas pertenecientes a conglomerados de mortalidad alta y las áreas pertenecientes a conglomerados de mortalidad baja.

Resultados. Se detectaron cinco conglomerados de mortalidad alta entre el 21 de marzo y el 27 de agosto en el Aglomerado Gran Buenos Aires (AGBA) y noreste de la provincia de Buenos Aires. Los conglomerados de mortalidad baja se localizaron en la periferia del AGBA, desde mediados de septiembre a mediados de octubre, y en el centro y noroeste de la Argentina, entre fines de abril y fines de agosto. Los conglomerados de mortalidad alta se localizaron en áreas con mayor densidad poblacional y mayor porcentaje de población adulta mayor en comparación a los conglomerados de mortalidad baja.

Conclusiones. No se detectaron conglomerados de mortalidad alta entre septiembre y mediados de octubre. Tampoco hemos detectado una difusión espacial de muertes hacia áreas de nivel socioeconómico bajo a nivel nacional. Nuestros resultados apoyan el modelo de difusión de la mortalidad en una primera fase, que afecta a la principal área urbana de la Argentina.
\end{abstract}

Palabras clave Análisis espacio-temporal; agrupamiento espacio-temporal; infecciones por coronavirus; mortalidad; Argentina.

La mortalidad por COVID-19 aumenta a un ritmo sostenido en América, totalizando 708996 fallecimientos al 26 de noviembre de 2020 (1), representando un 50\% del total de muertes a nivel mundial. En este contexto, la Argentina ha mostrado un aumento importante en el número de muertes diarias, durante los últimos meses, que la ha posicionado como el segundo país con mayor tasa de mortalidad por COVID-19 de América(1). Hasta el mes de mayo la mortalidad diaria por COVID-19 no superaba los 20 fallecimientos en Argentina, mientras que a principios de septiembre este número ya superaba las 200 muertes
(2). Este aumento de la mortalidad podría no ser homogéneo ya que existe una disparidad alta en el riesgo de mortalidad entre las jurisdicciones de la Argentina y al interior de la provincia de Buenos Aires. La Ciudad Autónoma de Buenos Aires (CABA), Jujuy y Buenos Aires son las jurisdicciones con mayores tasas de mortalidad en Argentina, pero el Área Metropolitana de Buenos Aires, la mayor aglomeración urbana de la Argentina compuesta por partidos de la provincia de Buenos Aires más la CABA, presenta la segunda tasa más alta comparada a todas las jurisdicciones del país (2).

\footnotetext{
1 Departamento de Salud Comunitaria, Consejo Nacional de Investigaciones Científicas y Técnicas (CONICET), Universidad Nacional de Lanús, Buenos Aires, Argentina.
} 
Estas desigualdades espaciales podrían ser el reflejo de desigualdades socioeconómicas. El riesgo de muerte por COVID-19 parece tener un mayor impacto en poblaciones socioeconómicamente más vulnerables. Estudios recientes muestran mayores tasas de mortalidad, incidencia y letalidad en la población afroamericana de los EEUU (3-5), y mayores tasas de mortalidad en áreas con mayor proporción de minorías étnicas y mayor proporción de población con ingresos bajos en Inglaterra (6). Estas desigualdades socioeconómicas parecen traducirse a diferentes escalas geográficas, desde variaciones geográficas al interior de las ciudades $(7,8)$ hasta variaciones al interior de los países (4). Sin embargo, otros estudios reportan mayores tasas de mortalidad en áreas de nivel socioeconómico alto $(9,10)$.

La incorporación de la dimensión espacial en el estudio de la mortalidad por COVID-19 permitiría plantear nuevas hipótesis acerca de los factores que contribuyen a la difusión del riesgo de mortalidad en Argentina. La difusión espacial puede definirse como la propagación de un fenómeno de un lugar a otro (11), e implica la interacción entre las dimensiones espacial y temporal. En nuestro caso, podemos plantear la existencia de interacción espacio-temporal cuando las muertes que se encuentran próximas en el espacio también tienden a estar próximas en el tiempo (12).

Desde la geografía, se ha planteado un modelo cualitativo de la difusión de la mortalidad al interior de un sistema de ciudades en el contexto de una pandemia por una enfermedad transmisible (13). Primero, las ciudades grandes experimentan los primeros picos de mortalidad y actúan como reservorios de infección. Segundo, esto provoca una transmisión jerárquica, de las ciudades grandes hacia ciudades más pequeñas. Tercero, debido al tamaño pequeño de las poblaciones susceptibles en las ciudades de menor tamaño, la recurrencia de sus picos de mortalidad es originada principalmente a través de la transmisión jerárquica. Con base en el modelo planteado y las investigaciones recientes sobre desigualdades socioeconómicas en la mortalidad por COVID-19, nos preguntamos de manera exploratoria: ¿la concentración espacio-temporal de muertes por COVID-19 ha mostrado una difusión de las áreas más densamente pobladas hacia áreas de menor densidad poblacional? ¿Esta difusión afectó primero a las áreas de nivel socioeconómico alto para luego afectar a áreas de nivel socioeconómico bajo?

Por lo tanto, los objetivos del presente estudio son: describir la difusión espacio-temporal de las muertes por COVID-19 y analizar sus desigualdades socio-espaciales en la Argentina.

\section{MÉTODOS}

Fue utilizada la información recopilada diariamente sobre casos sospechosos de COVID-19, mediante el Sistema Nacional de Vigilancia en Salud, y de acceso abierto por el Ministerio de Salud de la Nación (14). Debido a que el 65,9\% de las muertes de la Ciudad Autónoma de Buenos Aires (CABA) no contaba con el dato de la comuna de residencia, se utilizaron los datos de casos de COVID-19 publicados por el Gobierno de la Ciudad de Buenos Aires (15) para el análisis de la mortalidad en esta jurisdicción. Estos datos registraron un 4,6\% de muertes con comuna de residencia indefinida sobre un total de 4983 muertes por COVID-19 producidas al 17 de octubre de 2020 y con residencia en la CABA. Fuera de la CABA se registró un $0,4 \%$ de muertes sin departamento (partido en el caso de la provincia de Buenos Aires) de residencia sobre un total de 24004 fallecimientos al 17 de octubre de 2020. Del total de casos confirmados, usamos los datos referidos al momento (día, mes y año) y el lugar de residencia de las personas fallecidas hasta el 17 de octubre de 2020. En ambas fuentes de datos se utilizó la notificación de muertes actualizada al 14 de noviembre de 2020.

Se utilizó la técnica de escaneo espacio-temporal por permutaciones para detectar la presencia de conglomerados espacio-temporales de muertes por COVID-19 en Argentina durante el período del 3 de marzo de 2020 (fecha del primer caso confirmado) al 17 de octubre de 2020. Esta técnica detecta un conglomerado espacio-temporal si un área, durante un período de tiempo específico, tiene una proporción mayor de casos con respecto al resto de las áreas durante ese mismo período de tiempo (16). Para ejecutar el análisis espaciotemporal, es necesario contar tanto con la dimensión temporal como con la dimensión espacial de cada muerte. El tiempo fue considerado como el día de la muerte y el espacio geográfico como el departamento, partido o comuna de residencia. Al trabajar con las variaciones diarias de la mortalidad, es posible evaluar las variaciones espacio-temporales de la mortalidad prescindiendo de la población residente en cada área, ya que a esa escala temporal son muy poco probables los cambios bruscos de tamaño poblacional o de la estructura demográfica entre las unidades espaciales. Hay que agregar que estos cambios son mucho menos probables en el contexto de las diferentes medidas que han comprendido el Aislamiento Social Preventivo y Obligatorio (ASPO) en Argentina desde el 20 de marzo de 2020 (17). En total se consideraron 527 unidades espaciales, compuestas por departamentos, partidos y comunas. En nuestro estudio, la técnica de escaneo espacio temporal investigó la concentración de muertes dentro de una ventana de tiempo variable en diferentes áreas geográficas para comparar el número esperado de muertes y el número real de muertes dentro y fuera de la ventana (16). El tamaño de la ventana temporal y geográfica puede variar dentro de límites preestablecidos. Para nuestro análisis, la ventana de escaneo variable se estableció en un límite máximo del 10\% de las muertes y el 50\% del período de estudio. La elección del $10 \%$ buscó detectar conglomerados de menor extensión geográfica. El resultado es un conjunto de cilindros donde la base representa el área y la altura representa el período de tiempo en el que se concentran las muertes. Se calcularon las verosimilitudes máximas para determinar los conglomerados con menores probabilidades de ser explicados por el azar, cuyo valor de $P$ es asignado por el test de hipótesis de Montecarlo realizando 9999 replicaciones (16).

En segundo término, se calcularon pruebas de Wilcoxon de dos muestras para comparar una serie de características sociodemográficas, obtenidas del Censo 2010 (18), entre las áreas pertenecientes a conglomerados de mortalidad alta y las áreas pertenecientes a conglomerados de mortalidad baja. Se compararon tres variables: la densidad poblacional (habitantes por kilómetro cuadrado), como indicador del nivel de concentración poblacional; el porcentaje de hogares con Necesidades Básicas Insatisfechas (NBI), como un indicador de pobreza estructural; y el porcentaje de población con 60 o más años de edad, dado que la población de adultos mayores representa una de las de mayor riesgo de muerte (19).

El análisis de escaneo espacio temporal por permutaciones se llevó a cabo con el programa SaTScan v9.4.4 (16), mientras que 
las pruebas de Wilcoxon se calcularon con el programa Stata/ SE 13.1.

\section{RESULTADOS}

El escaneo espacio temporal por permutaciones detectó diez conglomerados estadísticamente significativos, de los cuales cinco tuvieron más muertes que las esperadas y los restantes cinco presentaron una mortalidad menor a la esperada (figura 1). Los conglomerados de mortalidad alta se clasificarían geográficamente en dos grupos; primero, dos conglomerados localizados en el noreste de la provincia de Buenos Aires incluyendo dos partidos de la periferia norte del Aglomerado Gran Buenos Aires (20) (AGBA: CABA más 30 partidos que, en conjunto, forman la mancha urbana o continuidad de viviendas urbanas conectadas por calles), siendo el conglomerado 9 el primero en emerger a fines de marzo; segundo, tres conglomerados localizados en comunas y partidos de alta densidad poblacional del AGBA entre principios de abril y mediados de agosto (figura 1: conglomerados 3, 5 y 6). Los conglomerados de mortalidad baja también pueden clasificarse en dos grupos: primero, tres conglomerados de mayor extensión geográfica, localizados en el centro y noroeste de la Argentina entre fines de abril y fines de agosto; segundo, dos conglomerados compuestos mayormente por partidos de la periferia del AGBA desde la segunda quincena de septiembre hasta la segunda quincena de octubre (final del período de estudio).

Finalmente, la figura 2 no muestra diferencias en las medianas del porcentaje de hogares con NBI entre áreas pertenecientes a conglomerados de mortalidad alta y conglomerados de mortalidad baja (prueba de Wilcoxon: $Z=-1,73 ; P=0,08$ ). Tanto la densidad poblacional como el porcentaje de población de 60 o más años mostraron medianas mayores en áreas pertenecientes a conglomerados de mortalidad alta con respecto a las áreas ubicadas en conglomerados de mortalidad baja (figura 2; prueba de Wilcoxon para la densidad poblacional: $Z=-7,61$; $P<0,0001$; prueba de Wilcoxon para el porcentaje de población de 60 o más años: $Z=-3,18 ; P<0,01)$.

FIGURA 1. Conglomerados espacio-temporales de mortalidad por COVID-19 (todos los conglomerados con valor $P<0,05)$. Argentina, 3 de marzo de 2020 - 17 de octubre 2020.

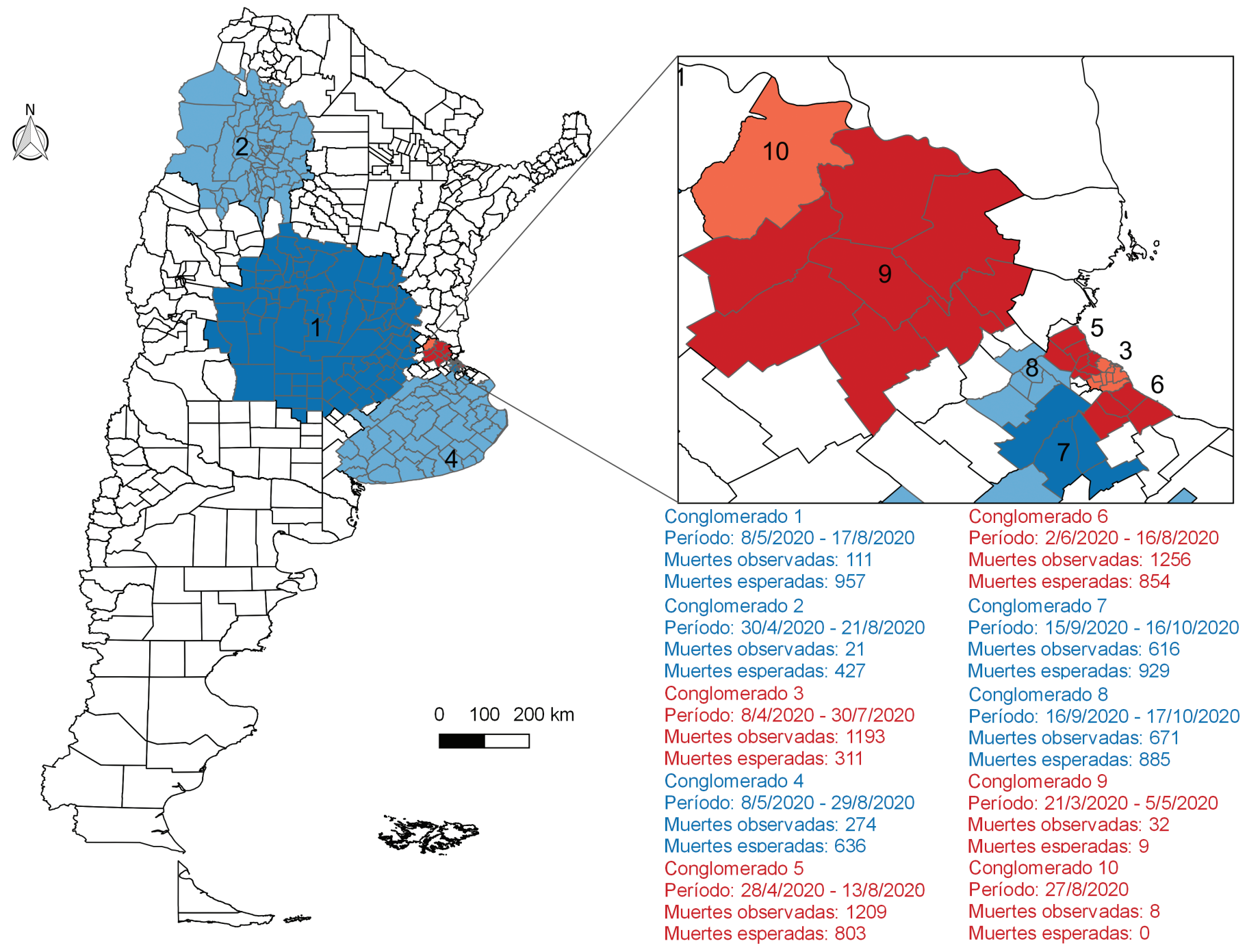


FIGURA 2. Distribución del porcentaje de hogares con Necesidades Básicas Insatisfechas (NBI, arriba), la densidad poblacional (centro) y el porcentaje de población de 60 o más años (abajo) entre áreas pertenecientes a conglomerados de mortalidad baja y áreas pertenecientes a conglomerados de mortalidad alta.

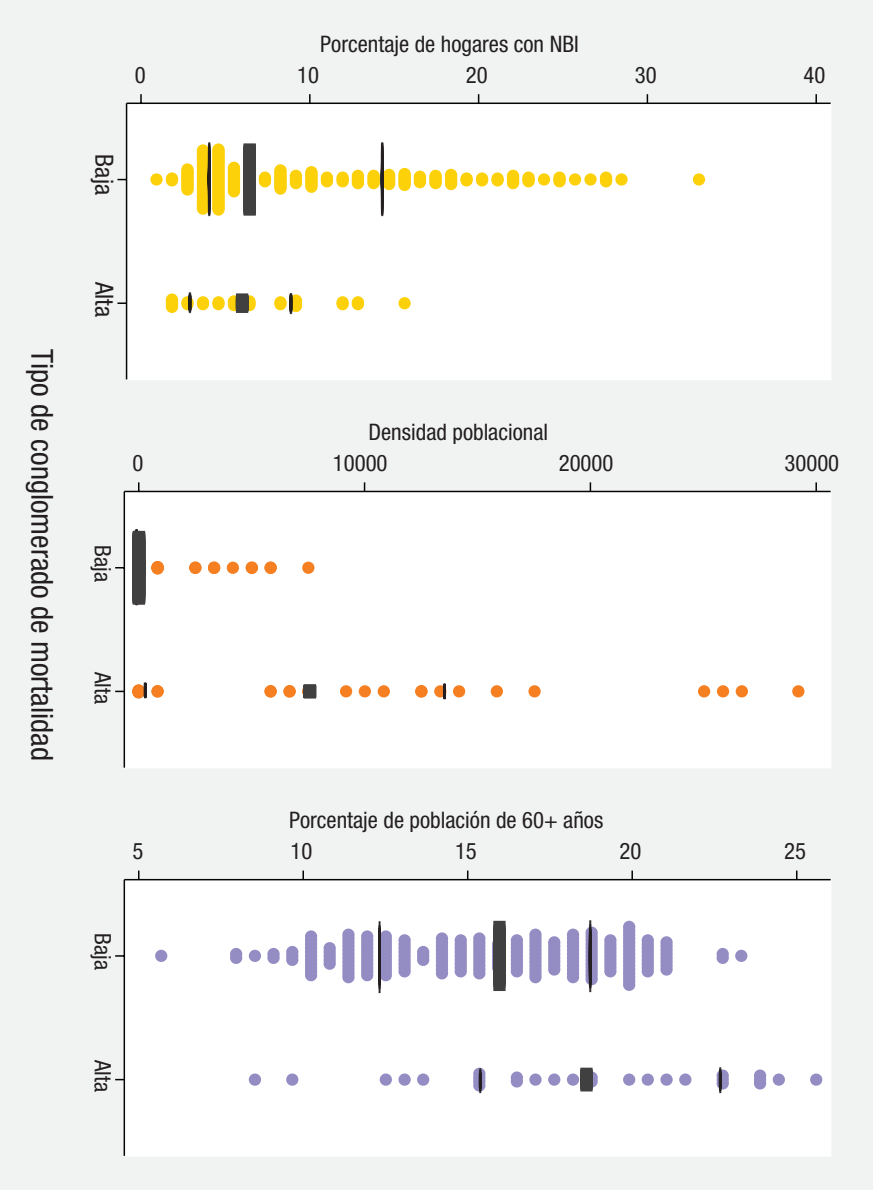

Leyenda: cada punto representa un área (comuna, departamento o partido), los rectángulos grises representan la mediana y las líneas grises representan el rango intercuartílico. Argentina, 2010, 2020.

\section{DISCUSIÓN}

El análisis espacio-temporal exploratorio realizado en este estudio permitió detectar conglomerados de mortalidad por COVID-19 en Argentina durante los primeros siete meses de circulación del virus en el país. Desde la primera muerte, producida el 7 de marzo de 2020, hasta el 17 de octubre de 2020, los conglomerados de mortalidad alta se detectaron entre el 21 de marzo y el 27 de agosto en el AGBA y noreste de la provincia de Buenos Aires. Este patrón espacio-temporal parece indicar la ausencia de la emergencia de conglomerados de mortalidad alta durante septiembre y principios de octubre. Sin embargo, estos resultados deben considerarse preliminares dado que los casos de COVID-19 han presentado una dispersión hacia el interior de la Argentina (21) durante este período.

Los conglomerados de mortalidad alta tendieron a presentar áreas con mayor densidad poblacional y mayor porcentaje de población de 60 o más años en comparación a los conglomerados de mortalidad baja. En el caso de la densidad poblacional, el resultado es esperable de acuerdo a lo planteado en la primera fase del modelo de difusión de la mortalidad. Con respecto al porcentaje de población de 60 o más años, lo observado concuerda con la tendencia global de un mayor riesgo de mortalidad por COVID-19 en población adulta mayor (19). Nuestros resultados difieren de los encontrados en otros países donde registraron un mayor riesgo de mortalidad en áreas de nivel socioeconómico bajo. En Estados Unidos se encontró un mayor riesgo de mortalidad en condados con mayor porcentaje de población en situación de pobreza (5), mientras que en Inglaterra este riesgo se acrecentó en áreas con mayor proporción de minorías étnicas y mayor proporción de población con ingresos bajos (6). En España la tasa de letalidad ajustada fue mayor en áreas de nivel socioeconómico bajo (22). En comparación a la Argentina, en Gran Bretaña y España hubo una mayor circulación del virus previa a la aplicación de medidas de distanciamiento social a nivel nacional. Al día de implementación de estas medidas, en Gran Bretaña ya se habían registrado 232 muertes por COVID-19 acumuladas $(1,23)$, mientras que España acumulaba 468 muertes (24). En Argentina, al día de implementación del ASPO se registraban 3 muertes (1). Es posible que una mayor circulación del virus previa a la aplicación de medidas de distanciamiento social en España y Gran Bretaña haya provocado una difusión más rápida hacia áreas de menor nivel socioeconómico. En la Ciudad de San Pablo (Brasil) el riesgo de mortalidad fue mayor en áreas de nivel socioeconómico alto en la semana epidemiológica 13 (del 22 al 28 de marzo de 2020), para luego pasar a ser mayor en áreas de nivel socioeconómico bajo (25). A diferencia de otros países sudamericanos, se estima que la Argentina ha sido uno de los países que más ha reducido la movilidad de su población durante los primeros meses de circulación del virus en el país (26). Esta mayor reducción ha estado aparentemente asociada a las medidas de cuarentena impuestas en algunos países, como el ASPO implementado en Argentina (26).

De acuerdo al modelo cualitativo de difusión de la mortalidad en el contexto de una pandemia (13), se esperaría un primer conglomerado de mortalidad alta en el área urbana principal de la Argentina. Si bien el primer conglomerado de mortalidad alta fue detectado mayoritariamente en partidos del noreste de la provincia de Buenos Aires, caracterizados por una red de ciudades pequeñas y medianas, también ocupó dos partidos de la periferia norte del AGBA. Esto, sumado a la emergencia de un conglomerado de mortalidad alta en la CABA dos semanas y media después, apoyaría parcialmente lo planteado por el modelo de difusión de la mortalidad en una primera etapa.

De los cinco conglomerados de mortalidad alta, tres (conglomerados 3, 5 y 6) fueron localizados en comunas y partidos de densidad alta del AGBA. Al observar la secuencia temporal de aparición de estos conglomerados, luego de la emergencia del conglomerado 3 en comunas de CABA (áreas de densidad poblacional alta) a principios de abril, emergió el conglomerado 5 en zona norte de la CABA y partidos contiguos al norte (Vicente López, San Isidro) a finales del mismo mes. Luego, a principios de junio, se detectó el conglomerado 6 en partidos localizados al sur de la CABA. Si se compara el porcentaje de hogares con NBI entre los conglomerados 5 (mediana $=2,4$; rango $=1,7-6,7)$ y 6 (mediana $=7,4 ;$ rango=5,0-9,3), existiría algún indicio de una difusión intra-urbana de la mortalidad hacia áreas de menor nivel socioeconómico hasta mediados de agosto. 
Sin embargo, esta difusión de la mortalidad no parece haber continuado hacia otros partidos periféricos del AGBA. Nuestro análisis muestra dos conglomerados de mortalidad baja en partidos de la periferia sur y oeste entre mediados de septiembre y mediados de octubre, caracterizados por presentar mayores ejes de pobreza con respecto a la CABA y partidos de la zona norte próximos (27). Esto es importante, ya que existen fuertes desigualdades socioeconómicas en los factores de riesgo de enfermedades crónicas de la población anciana urbana en América Latina (28). Los mayores valores del índice de masa corporal y la obesidad se concentraron en las poblaciones más pobres de la Ciudad Autónoma de Buenos Aires (29), y es probable que este patrón sea similar en poblaciones de nivel socioeconómico bajo del resto del AGBA.

Otro conjunto de conglomerados de mortalidad baja fue detectado en el centro y noroeste de la Argentina desde finales de abril hasta finales de agosto. La finalización de estos conglomerados coincide con la "federalización"(21) de la transmisión del virus fuera del AGBA hacia el resto de la Argentina a partir de septiembre. Sin embargo, el análisis espacio-temporal llevado a cabo en este estudio no da cuenta de un aumento mayor al esperado en el número de muertes fuera del AGBA hasta mediados de octubre, como sí lo predice la segunda fase del modelo de difusión de la mortalidad. Es posible que una combinación de políticas locales que restringen la circulación e interacción de personas (30-32), sumadas a medidas de auto-aislamiento por parte de poblaciones vulnerables (población con comorbilidades y población adulta mayor), hayan provocado una disminución de casos e impedido el posterior ascenso de la mortalidad en estos departamentos.

Este estudio presenta varias limitaciones. Primero, podría existir un retraso en la notificación de muertes en el interior de la Argentina (33) que podría traducirse en la no emergencia de conglomerados de mortalidad alta a partir de septiembre. Segundo, la notificación de los fallecimientos por jurisdicción se realiza teniendo en cuenta el lugar de residencia según el Registro Nacional de las Personas, por lo que aquellos cambios de residencia previos al fallecimiento por COVID-19 y no incorporados en este registro provocarían una asignación errónea de estas muertes en determinadas áreas. Tercero, el "problema de la unidad de área modificable" es propio del uso de áreas definidas arbitrariamente que pueden agregarse o desagregarse en otras áreas, lo que implica que se pueden obtener resultados diferentes si, sobre una misma área de estudio, se utilizan diferentes conjuntos de unidades espaciales. Cuarto, el uso de comunas/departamentos/partidos como unidades espaciales impide analizar las variaciones espacio-temporales de la mortalidad a escalas geográficas que logren captar diferenciaciones socioeconómicas intraurbanas o variaciones urbano-rurales de la mortalidad al interior de cada comuna/departamento/partido. Quinto, no contamos con información sobre la prevalencia de comorbilidades en las comunas/departamentos/partidos, por lo que no es posible determinar si la difusión de la mortalidad se produjo en áreas de mayor prevalencia de enfermedades crónicas y factores de riesgo asociados.

Por otro lado, este estudio presenta algunas fortalezas. Primero, el modelo espacio-temporal por permutaciones prescinde de la población en riesgo y puede ser utilizado a escalas temporales en las que no suelen existir datos de población (variaciones a nivel de días, semanas y meses) o en años alejados a la realización de censos poblacionales. Segundo, nuestro período de estudio incluye las 3459 registradas en la provincia de Buenos Aires, e incorporadas a partir del 25 de septiembre, por medio de una nueva metodología tendiente al registro en tiempo real de las muertes por COVID-19 (34). Además, debido a que en el resto de las jurisdicciones puede existir un retraso en el registro de fallecimientos, utilizamos datos actualizados al 14 de noviembre de 2020.

La conclusión principal de este estudio es la ausencia de conglomerados activos de mortalidad alta en Argentina durante septiembre y hasta el 17 de octubre de 2020, fin de nuestro período de estudio. Tampoco hemos detectado una difusión espacial de muertes hacia áreas de nivel socioeconómico bajo a nivel nacional. En concordancia con lo planteado en la primera fase del modelo de difusión de la mortalidad, los conglomerados de mortalidad alta fueron detectados, parcial o completamente, en el AGBA. Sin embargo, no se detectaron conglomerados de mortalidad alta en el resto del país, como sí era esperado en una segunda fase de difusión de la mortalidad. Nuestros resultados sugieren que las medidas implementadas en el marco del ASPO podrían haber disminuido la transmisión del virus y evitado la emergencia de conglomerados de mortalidad alta en áreas de nivel socioeconómico bajo y fuera de la AGBA, principal área urbana de la Argentina. Sin embargo, nuestro estudio exploró las variaciones espacio-temporales de las muertes por COVID-19 y determinadas características de área asociadas a la emergencia de los conglomerados, por lo que los resultados obtenidos no son concluyentes acerca del impacto de estas intervenciones sobre el territorio nacional.

Agradecimientos. A Betiana Menestrina, Lucas Leveau y Daniela Obando por el apoyo brindado en la realización de este estudio.

Contribución de los autores. CML contribuyó al diseño, análisis de datos, interpretación y redacción de los primeros y posteriores borradores del artículo.

\section{Conflicto de intereses. Ninguno declarado por el autor}

Declaración. Las opiniones expresadas en este manuscrito son responsabilidad del autor y no reflejan necesariamente los criterios ni la política de la RPSP/PAJPH y/o de la OPS.

\section{REFERENCIAS}

1. WHO. WHO Coronavirus Disease (COVID-19) Dashboard 2020. Disponible en: https://covid19.who.int. Acceso el 14 de agosto de 2020

2. Ministerio de Salud de la Nación. Boletín integrado de vigilancia (SE 40). Argentina: Ministerio de Salud de la Nación; 2020.
3. Chen JT, Waterman PD, Krieger N. COVID-19 and the unequal surge in mortality rates in Massachusetts, by city/town and ZIP code measures of poverty, household crowding, race/ethnicity, and racialized economic segregation. Harvard Center for Population and Development Studies; 2020. 
4. Zhang $\mathrm{CH}$, Schwartz GG. Spatial Disparities in Coronavirus Incidence and Mortality in the United States: An Ecological Analysis as of May 2020. J. Rural Health 2020;36:433-45.

5. Fielding-Miller RK, Sundaram ME, Brouwer K. Social determinants of COVID-19 mortality at the county level. PLOS ONE 2020;15:e0240151.

6. Rose TC, Mason K, Pennington A, McHale P, Taylor-Robinson DC, Barr B. Inequalities in COVID19 mortality related to ethnicity and socioeconomic deprivation. medRxiv 2020;

7. Cordes J, Castro MC. Spatial analysis of COVID-19 clusters and contextual factors in New York City. Spat. Spatio-Temporal Epidemiol. 2020;100355.

8. Bilal U, Barber S, Diez-Roux AV. Spatial Inequities in COVID-19 outcomes in Three US Cities. medRxiv 2020;2020.05.01.20087833.

9. Ehlert A. The socioeconomic determinants of COVID-19: A spatial analysis of German county level data. medRxiv 2020;

10. Maciel JAC, Castro-Silva II, Farias MR de. Initial analysis of the spatial correlation between the incidence of COVID-19 and human development in the municipalities of the state of Ceará in Brazil. Rev. Bras. Epidemiol. 2020;23:e200057.

11. Brown LA. Diffusion. En: International Encyclopedia of Human Geography. Elsevier; 2009. páginas 170-84.

12. Pike MC, Smith PG. Disease clustering: a generalization of Knox's approach to the detection of space-time interactions. Biometrics $1968 ; 541-556$.

13. Cliff A, Haggett P, Smallman-Raynor M. Deciphering global epidemics: analytical approaches to the disease records of world cities, 1888-1912. Cambridge University Press; 1998.

14. Ministerio de Salud de la Nación. Datos Abiertos del Ministerio de Salud - COVID-19. Casos registrados en la República Argentina. Disponible en: http://datos.salud.gob.ar/. Acceso el 8 de junio de 2020

15. Buenos Aires Ciudad. Buenos Aires Data - Casos COVID-19. Disponible en: https:/ / data.buenosaires.gob.ar/. Acceso el de septiembre de 2020

16. Kulldorff M. SaTScan user guide for version 9.6. Disponible en: https://www.satscan.org/cgi-bin/satscan/register.pl/SaTScan Users_Guide.pdf?todo=process_userguide_download. Acceso el 11 de diciembre de 2020

17. Boletín Oficial República Argentina - Aislamiento Social Preventivo y Obligatorio - Decreto 297/2020. Disponible en: https://www. boletinoficial.gob.ar/detalleAviso/primera/227042. Acceso el 21 de agosto de 2020

18. Instituto Nacional de Estadística y Censos. WebINDEC: Población / Censos. Disponible en: https://www.indec.gob.ar/nivel3_default. asp?id_tema_1=2\&id_tema_2=41 Acceso el 11 de noviembre de 2018

19. Lloyd-Sherlock P, Ebrahim S, Geffen L, McKee M. Bearing the brunt of covid-19: older people in low and middle income countries. BMJ 2020;m1052.

20. Instituto Nacional de Estadística y Censos. ¿Qué es el Gran Buenos Aires? Disponible en: https://www.indec.gob.ar/dbindec/folleto_ gba.pdf. Acceso el 28 de noviembre de 2020

21. Télam. La Argentina atraviesa una "federalización de la pandemia" de coronavirus. Disponible en: https://www.telam.com.ar/ notas/202009/517311-ministerio-salud-federalizacion-pandemiaargentina.html. Acceso el 28 de noviembre de 2020

22. Dahal S, Mizumoto K, Rothenberg R, Chowell G. Investigating spatial variability in COVID-19 pandemic severity across 19 geographic areas, Spain, 2020. Disponible en: http://medrxiv.org/lookup/doi /10.1101/2020.04.14.20065524. Acceso el 7 de mayo de 2020
23. Strict new curbs on life in UK announced by PM. BBC News.Disponible en: https://www.bbc.com/news/uk-52012432. Acceso el 20 de agosto de 2020

24. Jones S. Spain orders nationwide lockdown to battle coronavirus. The Guardian. Disponible en: https://www.theguardian .com/world/2020/mar/14/spain-government-set-to-order-nation wide-coronavirus-lockdown. Acceso el 20 de agosto de 2020

25. Bermudi PMM, Lorenz C, de Aguiar BS, Failla MA, Barrozo LV, Neto FC. Spatiotemporal dynamic of COVID-19 mortality in the city of São Paulo, Brazil: shifting the high risk from the best to the worst socio-economic conditions. Disponible en: arXiv preprint arXiv:2008.02322. Acceso el 20 de agosto de 2020

26. de Oliveira GLA, de Lima LC, Silva I, da Câmara Ribeiro-Dantas M, Monteiro KH, Endo PT. Medidas de distanciamento social e mobilidade na América do Sul durante a pandemia por COVID-19: Condições necessárias e suficientes?. Disponible en: arXiv preprint arXiv:2006.04985. Acceso el 28 de noviembre de 2020

27. Marcos M, Mera G. Distribución y diferenciación espacial de la población. Explorando la articulación entre nivel socioeconómico y condición migratoria en la Aglomeración Gran Buenos Aires, año 2001. En: IV Congreso de la Asociación Latinoamericana de Población. La Habana, Cuba: 2010.

28. Palloni A, McEniry M. Aging and health status of elderly in Latin America and the Caribbean: preliminary findings. J. Cross-Cult. Gerontol. 2007;22:263-285.

29. Fleischer NL, Roux AVD, Alazraqui M, Spinelli H. Social patterning of chronic disease risk factors in a Latin American city. J. Urban Health 2008;85:923.

30. La Capital. Salió el decreto que oficializa dónde siguen las medidas estrictas. La Capital. Disponible en: https://www.lacapital. com.ar/santa-fe/coronavirus-oficializan-cuales-son-los-departa mentos-santafesinos-que-siguen-medidas-estrictas-n2615996.html. Acceso el 30 de noviembre de 2020

31. Página12. Córdoba endurece las medidas de aislamiento por aumentos de casos de coronavirus | Seis departamentos vuelven a Fase 1. Página12. Disponible en: https://www.pagina12.com. ar / 298335-cordoba-endurece-las-medidas-de-aislamiento-poraumentos-de-. Acceso el 30 de noviembre de 2020

32. El tucumano. Las nuevas restricciones de circulación en Tucumán rigen desde este sábado. Disponible en: https://www.eltucumano. $\mathrm{com} /$ noticia / actualidad/267323/las-nuevas-restricciones-decirculacion-en-tucuman-rigen-desde-este-sabado. Acceso el 30 de noviembre de 2020

33. Giménez J. Paso a paso: cómo se registran los casos y las muertes por coronavirus. Chequeado. Disponible en: https://chequeado. com/el-explicador/como-se-registran-los-casos-y-las-muertes-porcoronavirus-en-la-argentina/. Acceso el 28 de noviembre de 2020

34. Télam. Destacan el trabajo de Buenos Aires para obtener cifras en tiempo real de muertos por coronavirus. Disponible en: https:// www.telam.com.ar/notas/202009/518733-provincia-buenosaires-datos-cifras-casos-coronavirus.html. Acceso el 30 de noviembre de 2020

Manuscrito recibido el 23 de agosto de 2020. Aceptado para su publicación, tras revisión, el 1 de diciembre de 2020. 


\section{Space-time spread of COVID-19 deaths in Argentina}

ABSTRACT Objectives. Describe the space-time spread of COVID-19 deaths and analyze its socio-spatial inequalities in Argentina.

Methods. COVID-19 deaths in Argentina as of October 17, 2020 were analyzed using data on day, month, and year, and place of residence. The space-time permutation scan method was used to detect the presence of space-time clusters. Poverty levels, population densities, and percentage of older adults in the population were compared for areas in high-mortality clusters and low-mortality clusters.

Results. Five high-mortality clusters were detected between March 21 and August 27 in the Greater Buenos Aires conurbation and the northeast of the province of Buenos Aires. Low-mortality clusters were located on the periphery of the urban area from mid-September to mid-October and in central and northwestern Argentina between late April and late August. High-mortality clusters were located in areas with higher population densities and higher percentages of older adults in population, compared to low-mortality clusters.

Conclusions. No high-mortality clusters were detected between September and mid-October. Nor have we detected a spatial spread of deaths to areas of low socioeconomic status at the national level. Our results support the first phase of the mortality spread model, affecting the largest urban area in Argentina.

Keywords Spatio-temporal analysis; space-time clustering; coronavirus infections; mortality; Argentina.

\section{Distribuição espaço-temporal de mortes por COVID-19 na Argentina}

Resumo Objetivo. Descrever a distribuição espaço-temporal de mortes por COVID-19 e analisar desigualdades socioespaciais na Argentina.

Métodos. As mortes por COVID-19 ocorridas na Argentina até 17 de outubro de 2020 foram analisadas a partir de dados referentes ao dia, mês e ano e local de residência. A estatística scan utilizando modelo de permutação espaço-tempo foi aplicada para detectar conglomerados espaço-temporais. Realizou-se a comparação do nível de pobreza, densidade populacional e percentual de população idosa entre as áreas pertencentes aos conglomerados com alta mortalidade e as áreas pertencentes aos conglomerados com baixa mortalidade.

Resultados. Cinco conglomerados com alta mortalidade foram detectados entre 21 de março e 27 de agosto na região da Grande Buenos Aires e no nordeste da província de Buenos Aires. Os conglomerados com baixa mortalidade estavam localizados na periferia da região da Grande Buenos Aires, de meados de setembro a meados de outubro, e nas regiões central e noroeste do país, entre o final de abril e final de agosto. Os conglomerados com alta mortalidade estavam localizados em áreas de maior densidade populacional e maior percentual de população idosa em comparação aos conglomerados com baixa mortalidade.

Conclusão. Não foram detectados conglomerados com alta mortalidade entre setembro e meados de outubro. Também não se observou a distribuição espacial de mortes em áreas com baixo nível socioeconômico em todo o país. Os resultados deste estudo respaldam o modelo de distribuição de mortes na primeira fase, atingindo a principal área urbana da Argentina.

Palavras-chave Análise Espaço-Temporal; Conglomerados Espaço-Temporais; Infecções por Coronavirus; Mortalidade; Argentina. 